
\title{
R Reserach S Suare \\ Identification of foodborne botulism with mixed serotypes A, B and $E$ and their in vivo profiles in four types of clinical specimens
}

Jianwei Xie ( $\nabla$ xiejwbmi@163.com )

\section{Research}

Keywords: Botulinum neurotoxin, Foodborne botulism, Serotype, Endopeptidase-mass spectrometry assay (Endopep-MS), Diagnostics, Clinical specimens

Posted Date: January 24th, 2022

DOI: https://doi.org/10.21203/rs.3.rs-1288396/v1

License: (c) (1) This work is licensed under a Creative Commons Attribution 4.0 International License. Read Full License 


\section{Abstract \\ Background}

Diagnosis of mixed botulism caused by multiple serotypes of botulinum neurotoxin (BoNT) is still a challenge due to the lack of reliable detection method. The present study develops a feasible laboratorial method to detect BoNTs in clinical samples and aims to provide insights into in vivo profiles of different toxin serotypes.

\section{Methods}

An isotope dilution Endopep-MS method for simultaneously detection of seven serotypes of BoNT was established and further applied to diagnosis of mixed botulism through one-step immunocapture procedure. A comprehensive analysis of four types of clinical specimens, i.e., serum, vomitus, urine, and gastric mucosa samples, from four suspected patients was performed.

\section{Results}

The established robust multiplex Endopep-MS method was applied to accurately identify and monitor BoNT/A, B and E in specimens during a clinically and therapeutically relevant timeframe. Serotypes and concentrations of BoNT in specimens revealed a good correlation with symptoms and progresses of disease. Additionally, serum was evidenced to be more suitable for detection BoNT/A with a detection window up to 12 days. Urine sample, although scarcely used in foodborne botulism diagnosis, was validated to be suitable for testing BoNTs with a longer detection window up to 25 days.

\section{Conclusions}

This is the first comprehensive analytical research on in vivo profiles of serotypes A, B and E in different types of specimens from mixed botulism cases to the best of our knowledge, and our method and findings facilitate the toxin detection and identification by clinical diagnostic laboratories.

\section{Introduction}

Botulinum neurotoxin (BoNT), a kind of proteolytic toxin produced by certain species of the genus Clostridium, particularly Clostridium botulinum, Clostridium butyricum and Clostridium baratii, is the most toxic biotoxin known to humankind that causes a paralytic illness called botulism [1-3]. Foodborne botulism, infant botulism and wound botulism are the most common poisoning types, which may eventually ends in respiratory failure and leads to death without immediate and appropriate treatment [4]. In addition, BoNT is well connected to a large variety of medical applications and intoxication incidents 
are on the rise [5]. Moreover, high toxicity and ease of production make BoNT a likely agent of bioterrorism [2].

Based on antigenic properties, BoNT can be divided into eight serotypes: A to G and FA. Serotypes A, B, E, and occasionally $\mathrm{F}$ give rise to botulism in human, $\mathrm{C}$ and $\mathrm{D}$ are commonly associated with botulism of animals, and type $G$ was usually isolated from soils [5-7]. BoNT/FA is a novel serotype discovered in 2016 with a hybrid-like structure of BoNT/A1 and BoNT/F5 [8]. As a $150 \mathrm{kDa}$ protein containing a heavy chain (HC, $100 \mathrm{kDa}$ ) and a light chain (LC, $50 \mathrm{kDa}$ ) connected by a disulfide bond [9, 10], BoNT has a zincdependent metalloprotease activity that cleaves SNARE (soluble N-ethylmaleimide-sensitive factor attachment protein receptor) proteins in peripheral neurons to block the release of neurotransmitters [11]. SNARE is a complex that includes three proteins: VAMP (vesicular-associated membrane protein), SNAP25 (synaptosomal-associated protein of $25 \mathrm{kDa}$ ) and syntaxin. Each BoNT serotype cleaves SNARE substrate at a distinct cleavage site [12]. BoNT/A, C and E each cleave SNAP-25 at unique sites of Gln 197-Arg 198, Arg 198-Ala 199 and Arg 180-lle 181 [13, 14]. BoNT/C also cleaves syntaxin. BoNT/B, D, F and $G$ each cleave VAMP at unique sites of Gln 76-Phe 77, Lys 59-Leu 60, Gln 58-Lys 59, and Ala 81-Ala $82[15-17]$.

Serotype differentiation and identification of BoNT is crucial for diagnosis of botulism, especially for mixed botulism, which is also helpful for epidemiological investigations and source tracing in a timely manner [18]. Furthermore, serotype differentiation can be used to identify novel serotypes or chimeras of BoNT [19]. The incubation period, symptoms and treatment are varied with serotypes. The onset symptom of BoNT/E is usually within $24 \mathrm{~h}$, whereas the incubation periods of BoNT/A (0-7 days) and BoNT/B (0-5 days) are much longer [20, 21] even in some cases up to 10-15 days after ingestion the contaminated food. Timely administration of serotype-specific antitoxin is an effective way to prevent the progression of neurological syndrome and shorten the duration of supportive treatment at early stage. However, differences in the time of presentation and confused symptoms caused by different serotypes can make clinical diagnosis difficult. Rapid and reliable laboratorial detection methods in biologic samples are necessary to support clinicians in rapid diagnosis and to help patients receive timely serotype-specific treatment.

To date various laboratory detection methods have been developed. Mouse bioassay is recognized as the "gold standard" for BoNT detection with a sensitivity of $1 \mathrm{U} / \mathrm{mL}$ (approximately $10 \mathrm{pg} / \mathrm{mL}$ ) [20, 22] but with a shortage of time-consuming and usage of animals. Immunological methods like lateral flow assays (LFAs) are commonly used for the advantages of low cost and easy operation [23]. Nonetheless, they are unable to determine the toxin serotype and activity simultaneously [20]. Recently, mass spectrometry-based method has been applied increasingly in toxin detection, owing to its high sensitivity and specificity. Endopeptidase-mass spectrometry (Endopep-MS) method which is based on the specific endopeptidase activity of toxin was developed to determine the activity and serotypes of BoNT. Methods for detection BoNT/A, B, E and $F$ have been established by using matrix-assisted laser desorption ionization-time of flight mass spectrometry (MALDI-TOF-MS) or liquid chromatography tandem mass spectrometry combined with a multiple reaction monitoring technique (LC-MS/MS(MRM)) [24, 25]. Rosen 
et al. reported a multiplex method for determination of BoNT/A, B and E in mimic clinical samples, and each BoNT serotype is extracted separately using individual serotype-specific antibody from the spiked serum samples [26]. For detecting of all seven serotypes, only Boyer reported a MALDI-TOF-MS method using four peptides as substrates among which two serotypes share one common substrate [27]. Consequently, up to now there is no LC-MS/MS based multiplex method for simultaneously detection of seven serotypes from $A$ to $G$, and no method has been extensively verified in real clinical samples so that limited information is available about the in vivo profile of different serotypes in poisoning individuals.

Here, a stable isotope dilution Endopep-MS method for simultaneously detection of BoNT A-G was developed, and the optimal substrates of each serotype and their respective stable isotope labeled internal standards (IS) were selected. Peptide cleavage products and IS peptide of specific serotypes were detected by LC-MS/MS (MRM), which can make a precise differentiation and quantification of seven serotypes. Furthermore, for serotypes A, B and E that cause human botulism, an antibody simultaneously specific recognizing the three serotypes was used to easily enrich toxins in complex biological matrices. The established method was finally applied to the analysis of clinical poisoning samples and diagnosis of mixed BoNT/A, B and E foodborne botulism for four cases from one family. Serum, urine, vomitus and postmortem blood and gastric mucosa samples were comprehensively analysed. To our knowledge, this is the first and detailed study of multiple BoNTs in clinical samples by MS which provides insights into the toxicokinetics of toxin serotypes and helpful information for early diagnosis and medical treatment of botulism.

\section{Materials And Methods}

\section{Materials and reagents}

BoNTs were purified and preserved in our laboratory. All peptides were synthesized by Sangon Biotech (Shanghai, China), as shown in Table 1. Monoclonal antibody RE052 specific to BoNT/E and mAb013 simultaneously specific to BoNT/A, B and E serotypes were kindly provided by Professor Jiannan Feng. Dynabeads ${ }^{\circledR}$ Protein $\mathrm{G}$ at $30 \mathrm{mg} / \mathrm{mL}$ in phosphate buffered saline (PBS) was purchased from Thermo Fisher Scientific (NY, USA).

\section{Optimization of the LC-MS/MS (MRM) method}

The analysis was performed in positive mode on a QTRAP® (AB SCIEX, Framingham, USA) 5500 System combined with an ACQUITY UPLC (Waters, Worcester, USA) in MRM. The column used was a BioBasic ${ }^{\text {TM }}$ C18 (Thermo Scientific ${ }^{\text {TM }}$, Torrance, USA) column $(100 \mathrm{~mm} \times 1 \mathrm{~mm}, 5 \mu \mathrm{m})$. The mobile phase consists of A: $\mathrm{H}_{2} \mathrm{O}$ with $0.1 \%$ (vol/vol) formic acid and B: acetonitrile in a linear gradient. Gradient profile was $1 \% \mathrm{~B}$ held at 0 to $1 \mathrm{~min}$, linearly increased to $25 \%$ B over $7 \mathrm{~min}$, and then increased to $95 \%$ B over $2 \mathrm{~min}$, and held another $3 \mathrm{~min}$, for a total run time of $12 \mathrm{~min}$. The flow rate was $0.25 \mathrm{~mL} / \mathrm{min}$, and the injection volume was $10 \mu \mathrm{L}$. 
Synthetic product peptides of seven serotypes were used to determine the optimal MRM transitions of the cleavage products of BoNT A-G. Two separate transitions of each peptide were monitored by LC-MS/MS, as shown in table 1.

\section{Quantification of peptide products through isotopic dilution}

Peptide products were quantified using stable isotope-labeled internal standard. IS of each serotype was consistent with its corresponding $\mathrm{NH}_{2}$-terminal ( $\mathrm{N}$-terminal) or $\mathrm{COOH}$-terminal (C-terminal) product peptide but was labeled with ${ }^{13} \mathrm{C}$ and ${ }^{15} \mathrm{~N}$. Except the IS of BoNT/C employed the C-terminal product of BoNT/E owing to the poor chromatographic retention of its C-terminal product. The ratio of analyte peak area relative to the corresponding internal standard (IS) was used to quantify the product peptides, which denoted the amount of BoNT.

The method validation follows the Guidance for Industry Bioanalytical Method Validation of US FDA. The calibration curve, limit of detection (LOD), lower limit of quantification (LLOQ), precision and recovery of all product peptides were assessed.

\section{Clinical sample analysis}

Four people from one family, a middle-aged man, his parents, and his son, ate vacuum-packed Spanish mackerel, chicken claws, and ham sausage purchased from local retail store accompanied with homemade pickled Chinese cabbage. The four people were designated as patients 1-4, as shown in Table 2. Patient 1 and patient 4 ate more food than the other two patients. The four patients felt no special discomfort at that time. After $14 \mathrm{~h}$, they all presented with nausea, vomiting, abdominal distension, fatigue and dizzy. The symptom of patient 4 was the most severe, who died from respiratory failure within $48 \mathrm{~h}$ in local clinic without antitoxin therapy or mechanical ventilation. LFA strips for BoNT/A were used to preliminary test the vomitus samples of patients 1,2 and 3 on day 1 , all showing positive results (Additional file 1: Fig. S1). Therefore, the three patients received type A equine botulinum antitoxin (Lanzhou Institution of Biological Products, China) therapy for 10 days, however the therapeutic effect was not obvious. They also received trachea intubation and mechanical ventilation for 20 days, 26 days and 10 days respectively.

Serum samples were collected from patients 1, 2 and 3 on day 3 and days 6-13 (days of onset). The midmorning urine samples were collected on day 3 and on days 24-25. Vomitus samples were collected on day 1. Pericardial blood and gastric mucosa were collected from patient 4 . Serum, urine and vomitus samples were centrifuged to separate supernatant for detection. Gastric mucosa was added with $500 \mu \mathrm{L}$ PBS and 7-mm stainless steel beads to homogenize by a TissueLyser (Schneider Elect, Rueil, France) for 3 min, and after centrifugation the supernatant was collected for enrichment and LC-MS/MS (MRM) analysis.

\section{BoNT enrichment and Endopep-MS reaction}


Monoclonal antibody simultaneously specific to BoNT/A, B and E serotypes was immobilized on Dynabeads Protein $\mathrm{G}$ to enrich toxins from samples. An aliquot of $30 \mu \mathrm{L}$ antibody-coated magnetic beads was mixed with $1 \mathrm{~mL}$ clinical samples by gently rotating for $2 \mathrm{~h}$ at RT to capture BoNT. Negative control was serum or urine from healthy donors while positive control was serum or urine from healthy donors spiked with $2 \mathrm{U}$ corresponding BoNT (Additional file 1: Fig. S2). Then the beads were removed and washed three times with $500 \mu \mathrm{L}$ PBS-Tween buffer (PBS with 0.05\% Tween-20), and transfer to another new EP tubes at the last wash. BoNT-captured immunobeads were incubated with a reaction buffer which contained $50 \mathrm{mM}$ Hepes (pH 7.4), $10 \mathrm{mM}$ dithiothreitol, $10 \mu \mathrm{M} \mathrm{ZnCl}_{2}$, IS of each serotype at different concentrations, $1 \mathrm{mg} / \mathrm{mL}$ bovine serum albumin (BSA) and $0.05 \mathrm{mM}$ peptide substrate mixtures $(0.05$ $\mathrm{mM} A P, \mathrm{BP}$ and $\mathrm{EP})$. All samples were incubated at $37^{\circ} \mathrm{C}$ for $5 \mathrm{~h}$. The incubation supernatant was collected and added with $5 \mu \mathrm{L} 10 \%$ TFA to stop the reaction. The solution was centrifuged at $14,000 \times g$ for 5 min prior to MS analysis.

\section{Results}

\section{LC-MS/MS (MRM) method}

An Endopep-MS method was constructed for simultaneously detection of BoNT/A to G. Seven peptide substrates, AP-GP, were employed to mimic the targets in vivo of BoNT/A to G respectively. Meanwhile, the cleavage products including $\mathrm{N}$-terminal (such as AP-N) and C-terminal (such as AP-C) peptides of corresponding substrates were also synthesized, as shown in Table 1. The C-terminal product of BoNT/C has strong polarity and the retention on reversed-phase columns is poor, therefore only CP-N (N-terminal) was included in the method. Tandem mass spectrometry was performed by monitoring the precursor to product ion transitions of each peptide under individually optimized conditions. Identification of each product peptides included retention time and precursor to product ion multiple reaction monitoring transition match. For example, the doubly charged ion $(714.1 \mathrm{~m} / \mathrm{z})$ of AP-N fragmenting to $585.4 \mathrm{~m} / \mathrm{z}$ and $261.2 \mathrm{~m} / \mathrm{z}$ were monitored, the doubly charged ion $(499.8 \mathrm{~m} / \mathrm{z})$ fragmenting to $491.3 \mathrm{~m} / \mathrm{z}$ and triply charged ion $333.5 \mathrm{~m} / \mathrm{z}$ fragmenting to $288.2 \mathrm{~m} / z$ of AP-C were monitored. The LC-MS/MS (MRM) peak intensities for $\mathrm{N}$ - and $\mathrm{C}$-terminal cleavage products and their respective IS peptides of BoNT A-G were shown in Fig. 1, using synthetic cleavage products of BoNT A to $G$ at medium concentrations of the calibration curve range.

\section{Performance of Endopep-MS method}

The LODs and LLOQs of all product peptides were assessed with a standard solution of $10 \mu \mathrm{g} / \mathrm{mL}$, followed by serial dilution until the signal to noise (S/N) exceeds 5 (LOD) and 10 (LLOQ). The working range starts from the LLOQ and to maximize the linearity of the method. At least six concentration levels for each product peptide were consisted in the calibration curves, and it was built by fitting the concentration of the peptides versus the peak area ratios of the peptide to IS with linear regression (Additional file 1: Fig. S3). The linear regression coefficient $R^{2}$ was $0.990-0.999$ for all peptides. Results of method validation were shown in Table S1 (Additional file 1). The precision was measured using six 
replicates respectively at low, medium and high concentrations of peptide standard solutions, resulting in RSD for all samples lower than $15 \%$. The recovery at three different concentrations was within $86 \%-121 \%$ (Table S2 in Additional file 1). The concentration and peak area ratios of peptide to IS of FP-N did not exhibit linear trend hence we exclude it in our method.

\section{Endopep-MS method for complex matrix sample}

Human botulism is commonly caused by BoNT/A, B and E, of which type A exerts the strongest toxicity and causes the most severe symptoms. Here an Endopep-MS method for clinical sample analysis was constructed using a monoclonal antibody specific to BoNT/A, B and E. We took BoNT/A as an example for method validation in complex matrix. Peptide AP, optimized from five different substrates (data not shown), was determined as the substrate of BoNT/A here due to the high enzymatic cleavage efficiency and relatively high mass spectrometry response of its product peptides. The LOD for BoNT/A spiked in PBS was $0.6 \mathrm{U} / \mathrm{mL}$ (Additional file 1: Fig. S4) defined by the toxin of which the product signal-to-noise ratio $(\mathrm{S} / \mathrm{N})$ exceeded three times the product of blank. The signal of $A P-C(S / N=21)$ at $0.6 \mathrm{U} / \mathrm{mL}$ of the toxin exceeded 3 times the signal detected in the blank (PBS) sample ( $\mathrm{S} / \mathrm{N}=4.3$ ). Fig. S5 (Additional file 1) showed the amount of cleavage product AP-N and AP-C under different concentrations of BoNT/A spiked into the Endopep-MS reaction mixture without enrichment. The $A_{\text {product }} / A_{I S}$ represents the ratio of peak area of product peptide to the internal standard.

The LOD of the Endopep-MS for BoNT/A was $0.5 \mathrm{U} / \mathrm{mL}$ in serum (Additional file 1: Fig. S6), equivalent to that of MALDI-TOF MS method using the same substrate, AP [28]. The amount of cleavage product AP-N and AP-C under different concentrations of BoNT/A was shown in Fig. S7 (Additional file 1). The linearities for BoNT/A in PBS and serum were shown in Figs. S8-9 (Additional file 1). As for peptides BP and EP, they have been validated in Rosen's work $[14,29]$ thus mainly the LODs were demonstrated here. Their sensitivities under the presence of corresponding BoNT/B and BoNT/E in serum were $1.5 \mathrm{U} / \mathrm{mL}$ and $1.8 \mathrm{U} / \mathrm{mL}$ (Additional file 1: Fig. S10). Three substrate peptides AP, BP and EP had no cross reactivity to each other under the presence of corresponding BoNTs.

\section{BoNT/A, B and E detection in clinical samples}

\section{Blood samples}

For serum samples of patients 1-3 collected on day 3 from symptom onset, only patient 2 was detected positive for BoNT/A (Table 3). While on day 6 , all sera of these three patients showed positive for BoNT/A, meanwhile patient 2 also showed positive for BoNT/E. The concentration of BoNT/A in the serial serum samples of individual patient showed a highest level on day 6 . For example, the concentration of BoNT/A was maximum on day 6 in the sera of patient 2 and decreased gradually from day 6 to day 12, as shown in Fig. 2. Chromatogram of cleavage products in blank serum and serum sample of patient 2 collected on day 3 was shown in Fig. 3a, b.

\section{Urine samples}


For patient 1, the urine was positive for BoNT/A, B and E (Fig. 3c, d) on day 3, still positive for both BoNT/A and E on day 24, and only positive for BoNT/A on day 25. Contrast to the relative high level of toxins in the urine sample on day 3 , BoNT/B and E were negative on day 25. For patient 2, the urine taken on day 3 was positive for both BoNT/A and E and only positive for BoNT/A on days 24-25. For patient 3, the urine of day 25 was positive for BoNT/E (Table 4).

\section{Vomitus samples}

The vomitus samples were only collected on day 1 . For patient 1 , it was positive for BoNT/A, B and $E$ simultaneously, as shown in Fig. 3e. For patients 2 and 3, it was only positive for BoNT/E (Table 4). It was worth noting that the detected BoNT/E level of patient 1 was higher than those of patient 2 and 3 , which was about twenty to thirty times of the latter.

\section{Postmortem samples}

Pericardial blood and gastric mucosa were collected from the deceased individual (patient 4). The pericardial blood sample was positive for BoNT/A despite haemolysis. The gastric mucosa was positive for BoNT/E (Table 4). Fig. 3f showed the N-terminal cleavage product of BoNT/E.

\section{Discussion}

Botulism is a life-threatening disease in humans and animals, which is caused by active BoNT. Timely diagnosis and treatment of botulism is crucial for survival due to the extreme potency and short onset time like E serotype [20], which relies on the laboratory confirmation. In addition, epidemiological investigation after an outbreak and source tracing also relies on the establishment of detection method [18]. In China, conventional laboratory procedures for botulism confirmation are bacterial culturing, LFA and mouse bioassay $[30,31]$. However, bacteria isolation and culturing detect $C$. botulinum rather than the active toxin thus is not sufficient to confirm the clinical diagnose, and the whole procedure takes at least seven days [31]. Mouse bioassay can detect active BoNT in contrast to PCR assay, however, it usually takes 1-4 days to complete and need live animals. Here a convenient multiplex Endopep-MS assay was established, which can make an activity determination and serotype differentiation of BoNT AG.

In our method, seven synthetic peptides were used to imitate the substrates in vivo of BoNT A-G. Substrate AP has been applied to Endopep-MS assay for BoNT detection by MALDI-TOF MS [28], but it has not been used by LC-MS/MS (MRM). BP and EP have been validated both in a single and multiplex Endopep-MS assay [14, 32]. CP and GP were the optimized peptide substrates in Wang's work for BoNT/C and $G$, respectively, which were validated in the MALDI-TOF MS method $[15,17]$. Product peptides of BoNT A-G were quantified using stable isotope dilution LC-MS/MS method, of which the validation results met the methodological requirements. The narrow linear range and poor mass response of the $\mathrm{N}$-terminal product peptides of serotype B, D, F and G are probably due to the large molecular weight and more acidic 
amino acids, while their respective $\mathrm{C}$-terminal peptide performs well. Presence of product peptide at either $\mathrm{N}$ - or C-terminal in sample was recognized as a positive result for BoNT.

Human botulism is commonly caused by BoNT/A, B and E, and recently mixed serotypes occurred increasingly worldwide, so we further focused on the establishment of a method to simultaneously detect BoNT/A, B and E in biological matrix. The common clinical samples for diagnose of botulism include serum, stool and gastric contents (for foodborne botulism). The complexity of these samples poses a challenge to the BoNT detection. Here, we employed the BoNT specific antibody-coated beads to extract BoNTs from clinical samples. In Rosen's work, serotypes A, B and E were extracted separately using the mono-serotype-specific antibodies [29]. In our work, the usage of antibody mAb013, a monoclonal antibody simultaneously specific to BoNT/A, B and E, can simplify the enrichment procedure and thus shorten the analysis time. Under the optimized immunocapture multiplex Endopep-MS method, the sensitivity for BoNT/A was $0.6 \mathrm{U} / \mathrm{mL}$ in PBS and $0.5 \mathrm{U} / \mathrm{mL}$ in spiked serum. The sensitivity of our method was in the same order of magnitude as reported by Rosen, et al [29]. LODs of BoNT/B and E in spiked serum were $1.5 \mathrm{U} / \mathrm{mL}$ and $1.8 \mathrm{U} / \mathrm{mL}$ respectively, both exhibited a good sensitivity and specificity.

Importantly, the method was successfully used to detect complex samples from suspected patients. Despite reports of poisoning cases involving multiple serotypes, there is no report on the detailed in vivo profiles of toxins involving serotypes, concentrations, detection window and preferred distribution. A comprehensive study of suspected samples was conducted and the mixed botulism case timeline was presented (Fig. 4, Tables 3-4). For patient 1, the urine sample taken on day 3 and vomitus sample taken on day 1 were both positive for BoNT/A, B and E. In these two samples, the level of BoNT/E was the highest among the three serotypes, and it was more than 10 times higher in patient 1 than in patient 2 and 3. The concentration of BoNT/A in urine was about three times higher than that for patient 2 , and the urine samples taken on days 24 and 25 were also positive for BoNT/A. Meanwhile, BoNT/B was only detected in the urine sample of patient 1 . Symptoms and the period of ventilation of patient 1 were more severe and longer than patient 3 , which was consistent with our detection results that both concentrations of BoNT/A and E of patient 1 were higher than those of patient 3.

For patient 2, serum samples were positive for BoNT/A on days 6-12, with a wider detection window versus patient 1 and patient 3 (day 9), and the level of BoNT/A in serum on day 6 was also the highest among three patients. Vomitus sample collected on day 1 was only positive for BoNT/E. Urine sample showed a positive result in BoNT/A and E on day 3, and it was still positive for BoNT/A on day 25. The duration of mechanical ventilation for patient 2 was the longest among three patients, due to her uncooperative with treatment and poor health condition. Unfortunately, patient 2 deceased two months after the discharge, mainly due to high blood pressure combined with diabetes and other diseases, and may also be due to the incomplete removal of BoNT from the body.

For patient 3 , serum sample was positive for BoNT/A on day 6 and turned to negative on day 10 . Vomitus sample was only positive for BoNT/E, and urine sample taken on day 25 was positive for BoNT/E. Symptoms of patient 3 were the mildest and his recovery was the fastest compared with the other two 
patients, which was consistent with the result that the level of BoNT/A in serum was the lowest among the three patients. The fast recovery may also due to his strong physical condition.

For patient 4, results indicated that BoNT/A was positive in serum, and BoNT/E was detected in gastric mucosa samples. The food eaten by patient 4 was far more than that taken by patients 2 and 3 , therefore, it's more likely that the amount of BoNT ingested by patient 4 was more than the other patients. As a result, patient 4 presented the most significant symptoms and unfortunately ended in respiratory failure.

Four categories of clinical samples from the four patients were analysed using our method. We observed that all serum samples were positive only for BoNT/A except one sample of patient 2 on day 6 was also positive for BoNT/E. Our observation coincided with the finding of Woodruff et al. that samples from patients with type $A$ botulism tend to be positive in serum than those from patients with types $B$ or $E$ botulism [20]. Results also indicated that serum is especially suitable as samples for BoNT/A botulism confirmation with a detection window up to 12 days (Fig. 4) but may not suitable for other serotypes. Vomitus sample is a valuable sample during the early stage of a foodborne intoxication, which provides an original source reflecting the BoNT ingestion. Urine sample is rarely reported to be used in laboratory confirmatory assays for botulism, however our results indicated that urine sample is suitable for testing multiple serotypes of BoNT and may provide a longer detection window up to 25 days. These findings provide important data for forensic investigation of BoNT poisoning.

The onset time of above four patients was about $14 \mathrm{~h}$ after the dinner, which was consistent with the feature that BoNT/E has the shortest incubation period among serotypes $A, B$, and E. BoNT/E exerts toxicity more quickly than serotype $A$ and $B$, due to its fast speed to enter neurons and to block the neurotransmission [33]. The fatality rate in patients with type $E$ foodborne botulism was reduced after receiving appropriate antitoxin treatment [34]. Therefore, individuals who were exposed to BoNT/E should be admitted to hospital and received antitoxin therapy as early as 24 hours after ingestion of contaminated food. Patient 4 with the most significant symptoms was misdiagnosed as acute gastroenteritis in the local clinic hence missing the optimal treatment window.

The main symptoms of four patients were autonomic dysfunction like nausea, vomiting, dizzy and fatigue, which were in accordance with the characteristics of BoNT/E intoxication [20]. Botulism from BoNT/A generally results in more severe disease presented as bulbar nerve dysfunction and skeletal muscle impairment such as dysphagia, diplopia and dyspnea, which usually requiring mechanical ventilation up to several months $[35,36]$. In our case, three patients all required relative short ventilation support treatment for 10-30 days. It's worth noting that there were no dysphagia and blurred vision presented on these patients, which occurred significantly more frequently in patients with type $A$ botulism [35]. The non-obvious symptoms implied that three patients were not mainly intoxicated by BoNT/A. Combining with the experimental results of Endopep-MS and the fact that recovery from serotype $E$ is faster than serotype $A$ [37], we considered the patients were intoxicated mainly by serotype $E$ accompanied with serotype $A$ or both $A$ and $B$. 
In order to further validate the serotype E-dominated results, E serotype specific mAb RE052 was used to capture the vomitus and urine samples in which BoNT/E showed positive when using mAb013. The results were consistent with those of $\mathrm{mAb013}$. It also made an explanation for the non-obvious therapeutic effect of three patients receiving type A equine botulinum antitoxin after detecting BoNT/A by LFA (Additional file 1: Fig. S1). In addition, vomitus samples were cultured for $C$. botulinum which only showed serotype E-positive. Together, it demonstrated that LFA cannot accurately differentiate the serotypes of BoNT while culturing may be more prone to identify the dominant growing strains and overlook some non-dominant strains, in turn highlighting the specificity and efficiency of the multiplex Endopep-MS method, especially in the diagnosis of mixed botulism.

Mixed botulism may be caused by different individual strains producing different types of toxins, or by certain rare strain which known to produce more than one serotype. For example, BoNT/A2, F4 and F5 were produced by a $C$. botulinum strain named Af84 [38]. In a case of infant botulism, $C$. botulinum type Af has been isolated and cultured from the stool sample [39]. However, here specimens were handled without further anaerobic isolation and culturing except vomitus samples, therefore we do not know the exact pathogenic strains and whether existing a multi-toxin-producing $C$. botulinum strain.

\section{Conclusions}

We developed a stable isotope dilution Endopep-MS method for serotype differentiation and activity determination of BoNT A-G, which is validated to meet the bioanalytical requirements. A method for serotypes A, B and $E$ detection in complex matrix using single antibody-coated magnetic beads was further constructed and successfully applied to poisoning clinical samples. Four cases of mixed botulism were determined by detection BoNT/A, B and $E$ in four types of clinical specimen in a clinically and therapeutically relevant timeframe, among which serotype E dominated. Detected serotypes and concentrations were consistent with clinical symptoms of four patients, demonstrating the multiplex Endopep-MS method has great significance to the timely and accurately diagnosis of botulism even trace to the source during an outbreak. This analytical method with high sensitivity and specificity can be a toxicological alternative or complementary to traditional forensic methods, and would be useful to elucidate how toxins distribute and circulate in vivo and offer a feasible way to clinical diagnosis of mixed botulism.

\section{Abbreviations}

BoNT

Botulinum neurotoxin

$\mathrm{HC}$

Heavy chain

LC

Light chain

SNARE 
Soluble N-ethylmaleimide-sensitive factor attachment protein receptor

VAMP

vesicular-associated membrane protein

SNAP-25

synaptosomal-associated protein of $25 \mathrm{kDa}$

LFAs

lateral flow assays

Endopep-MS

Endopeptidase-mass spectrometry

MALDI-TOF-MS

Matrix-assisted laser desorption ionization-time of flight mass spectrometry

LC-MS/MS(MRM)

Liquid chromatography tandem mass spectrometry combined with a multiple reaction monitoring technique

IS

Stable isotope labeled internal standards

PBS

Phosphate buffered saline

$\mathrm{N}$-terminal

$\mathrm{NH}_{2}$-terminal

C-terminal

$\mathrm{COOH}$-terminal

LOD

limit of detection

LLOQ

Lower limit of quantification

BSA

bovine serum albumin

$\mathrm{S} / \mathrm{N}$

signal-to-noise ratio.

\section{Declarations}

\section{Acknowledgements}

We are grateful to Professor Jiannan Feng for his kindly offer of monoclonal antibody RE052 and mAb013.

\section{Authors' contributions}


$\mathrm{HX}$ and JWX contributed to the idea and design of this research. JW performed the experiments and wrote the manuscript. HX designed the experiments and revised the manuscript. JC, BX and YJZ involved in the data analysis. CYW, XYL and XLH contributed to the resources of this research. All authors have read and approved the final manuscript.

\section{Funding}

This work was supported by the Key Project of National Key Research and Development Program (No. 2018YFC1602600).

\section{Availability of data and materials}

Not applicable.

\section{Declarations}

\section{Ethics approval and consent to participate}

The study was reviewed and approved by the Ethics Committee of Chinese PLA General Hospital.

\section{Consent for publication}

Not applicable.

\section{Competing interests}

The authors declare no conflict of interest.

\section{Author details}

${ }^{1}$ State Key Laboratory of Toxicology and Medical Countermeasures, Institute of Pharmacology and Toxicology, Academy of Military Medical Sciences, Beijing 100850, China. ${ }^{2}$ Poisoning Treatment Department, the Fifth Medical Center of Chinese PLA General Hospital, Beijing 100071, China. ${ }^{3}$ State Key Laboratory of Pathogen and Biosecurity, Institute of Microbiology and Epidemiology, Academy of Military Medical Sciences, Beijing 100071, China

\section{References}

1. Schiavo G, Matteoli M, Montecucco C. Neurotoxins affecting neuroexocytosis. Physiol Rev. 2000;80(2):717-66.

2. Arnon SS, Schechter R, Inglesby TV, Henderson DA, Bartlett JG, Ascher MS, et al. Botulinum toxin as a biological weapon: medical and public health management. JAMA. 2001;285(8):1059-70.

3. Smith TJ, Hill KK, Raphael BH. Historical and current perspectives on Clostridium botulinum diversity. Res Microbiol. 2015;166(4):290-302. 
4. Lonati D, Schicchi A, Crevani M, Buscaglia E, Scaravaggi G, Maida F, et al. Foodborne botulism: clinical diagnosis and medical treatment. Toxins. 2020;12(8):509-25.

5. Kroumpouzos G, Kassir M, Gupta M, Patil A, Goldust M. Complications of Botulinum toxin A: An update review. J Cosmet Dermatol. 2021;20(6):1585-90.

6. Woudstra C, Skarin H, Anniballi F, Fenicia L, Bano L, Drigo I, et al. Neurotoxin gene profiling of clostridium botulinum types $\mathrm{C}$ and $\mathrm{D}$ native to different countries within Europe. Appl Environ Microbiol. 2012;78(9):3120-7.

7. Sonnabend O, Sonnabend W, Heinzle R, Sigrist T, Dirnhofer R, Krech U. Isolation of Clostridium botulinum type $\mathrm{G}$ and identification of type $\mathrm{G}$ botulinal toxin in humans: report of five sudden unexpected deaths. J Infect Dis. 1981;(1):22-7.

8. Reynolds MH, Johnson KN, Schvaneveldt ER, Dewey DL, Hess SC. Efficacy of detection canines for avian botulism surveillance and mitigation. Conservation Science Practice. 2021;3(1):e397.

9. Maslanka SE, Lúquez C, Dykes JK, Tepp WH, Pier CL, Pellett S, et al. A Novel Botulinum Neurotoxin, Previously Reported as Serotype H, Has a Hybrid-Like Structure With Regions of Similarity to the Structures of Serotypes A and F and Is Neutralized With Serotype A Antitoxin. J Infect Dis. 2016;213(3):379-85.

10. Lacy DB, Tepp W, Cohen AC, Dasgupta BR, Stevens RC. Crystal structure of botulinum neurotoxin type A and implications for toxicity. Nat Struct Biol. 1998;5(10):898-902.

11. Swaminathan S, Eswaramoorthy S. Structural analysis of the catalytic and binding sites of Clostridium botulinum neurotoxin B. Nat Struct Biol. 2000;7(8):693-9.

12. Rossetto $O$, Pirazzini M, Montecucco $C$. Botulinum neurotoxins: genetic, structural and mechanistic insights. Nat Rev Microbiol. 2014;12(8):535-49.

13. Wictome S. Botulinum neurotoxins: mode of action and detection. J Appl Microbiol. 1998;84:87S97S.

14. Rosen O, Feldberg L, Gura S, Ran Z. Improved detection of botulinum type E by rational design of a new peptide substrate for endopeptidase-mass spectrometry assay. Anal Biochem. 2014;456(7):50-2.

15. Wang D, Krilich J, Baudys J, Barr JR, Kalb SR. Enhanced detection of type C botulinum neurotoxin by the Endopep-MS assay through optimization of peptide substrates. Biorg Med Chem. 2015;31(13):3667-73.

16. Shone CC, Quinn CP, Wait R, Hallis B, Fooks SG, Hambleton P. Proteolytic cleavage of synthetic fragments of vesicle-associated membrane protein, isoform-2 by botulinum type B neurotoxin. Eur $J$ Biochem. 1993;217(3):965-71.

17. Wang D, Baudys J, Hoyt K, Barr JR, Kalb SR. Sensitive detection of type G botulinum neurotoxin through Endopep-MS peptide substrate optimization. Anal Bioanal Chem. 2019;411:5489-97.

18. Thirunavukkarasu N, Johnson E, Pillai S, Hodge D, Stanker L, Wentz T, et al. Botulinum Neurotoxin Detection Methods for Public Health Response and Surveillance. Front Bioeng Biotechnol. 2018;6:80. 
19. Hansbauer EM, Skiba M, Endermann T, Weisemann J, Stern D, Dorner MB, et al. Detection, differentiation, and identification of botulinum neurotoxin serotypes $C, C D, D$, and $D C$ by highly specific immunoassays and mass spectrometry. Analyst. 2016;141(18):5281-97.

20. Woodruff BA, Griffin PM, Mccroskey LM, Smart JF, Wainwright RB, Bryant RG, et al. Clinical and laboratory comparison of botulism from toxin types A, B, and E in the United States, 1975-1988. J Infect Dis. 1992;166(6):1281-6.

21. Min M, Bai L, Peng X, Guo L, Wan K, Qiu Z. An Outbreak of Botulinum Types A, B, and E Associated With Vacuum-Packaged Salted Fish and Ham. J Emerg Med. 2021;60(6):760-3.

22. Ferreira JL. Comparison of amplified ELISA and mouse bioassay procedures for determination of botulinal toxins A, B, E, and F. J AOAC Int. 2001;84(1):85-8.

23. Notermans S, Nagel J. Assays for Botulinum and Tetanus Toxins. In: Simpson LL. Botulinum Neurotoxin: Academic Press, 1989:pp. 319-31.

24. Ching KH, Lin A, Mcgarvey JA, Stanker LH, Hnasko R. Rapid and selective detection of botulinum neurotoxin serotype-A and $-B$ with a single immunochromatographic test strip. J Immunol Methods. 2012;380(1-2):23-9.

25. Barr JR, Hercules M, Boyer AE, Woolfitt AR, Kalb SR, Antonis $P$, et al. Botulinum Neurotoxin Detection and Differentiation by Mass Spectrometry. Emerg Infect Dis. 2005;11(10):1578-83.

26. Wang D, Baudys J, Krilich J, Smith TJ, Kalb SR. A Two-Stage Multiplex Method for Quantitative Analysis of Botulinum Neurotoxins Type A, B, E, and F by MALDI-TOF Mass Spectrometry. Anal Chem. 2014;86(21):10847-54.

27. Boyer AE, Moura H, Woolfitt AR, Kalb SR, Mcwilliams LG, Pavlopoulos A, et al. From the mouse to the mass spectrometer: detection and differentiation of the endoproteinase activities of botulinum neurotoxins A-G by mass spectrometry. Anal Chem. 2005;77(13):3916-24.

28. Wang D, Baudys J, Ye Y, Rees JC, Barr JR, Pirkle JL, et al. Improved Detection of Botulinum Neurotoxin Serotype A by Endopep-MS through Peptide Substrate Modification. Anal Biochem. 2013;432(2):115-23.

29. Rosen O, Feldberg L, Yamin TS, Dor E, Barnea A, Weissberg A, et al. Development of a multiplex Endopep-MS assay for simultaneous detection of botulinum toxins A, B and E. Sci Rep. 2017;7(1):14859.

30. Ping Y, Dong, Wei W, Tao, Jiang, et al. Molecular and Epidemiological Characterization of Infant Botulism in Beijing, China. Biomed Environ Sci. 2017;30(6):460-4.

31. Xin W, Huang Y, Ji B, Li P, Wu Y, Liu J, et al. Identification and characterization of Clostridium botulinum strains associated with an infant botulism case in China. Anaerobe. 2019;55:1-7.

32. Rosen O, Feldberg L, Gura S, Zichel R. A new peptide substrate for enhanced botulinum neurotoxin type $B$ detection by endopeptidase-liquid chromatography-tandem mass spectrometry/multiple reaction monitoring assay. Anal Biochem. 2015;473:7-10.

33. Jiafu W, Jianghui M, Lawrence GW, Zurawski TH, Astrid S, Bodeker MDO, et al. Novel chimeras of botulinum neurotoxins $A$ and $E$ unveil contributions from the binding, translocation, and protease 
domains to their functional characteristics. J Biol Chem. 2008;283(25):16993-7002.

34. Mottate K, Yokote H, Mori S, Horita A, Miyatsu Y, Torii Y, et al. Retrospective survey to evaluate the safety and efficacy of Japanese botulinum antitoxin therapy in Japan. Toxicon Official Journal of the International Society on Toxinology. 2016;110:12-8.

35. Hughes JM, Blumenthal JR, Merson MH, Lombard GL, Dowell VR, Gangarosa EJ. Clinical features of types A and B food-borne botulism. Ann Intern Med. 1981;93(4):442-5.

36. Hellmich D, Wartenberg KE, Zierz S, Mueller TJ. Foodborne botulism due to ingestion of home-canned green beans: two case reports. J Med Case Reports. 2018;12(1):1-5.

37. Eleopra R, Tugnoli V, Rossetto O, De Grandis D. C. M. Different time courses of recovery after poisoning with botulinum neurotoxin serotypes A and E in humans. Neurosci Lett. 1998;256(3):1358.

38. Kalb SR, Baudys J, Smith TJ, Smith LA, Barr JR. Three enzymatically active neurotoxins of Clostridium botulinum strain Af84: BoNT/A2, /F4, and /F5. Anal Chem. 2014;86(7):3254-62.

39. De Jong LIT, Fernández RA, Pareja V, Giaroli G, Guidarelli SR, Dykes JK, et al. First Report of an Infant Botulism Case Due to Clostridium botulinum Type Af. J Clin Microbiol. 2015;53(2):740-2.

\section{Figures}

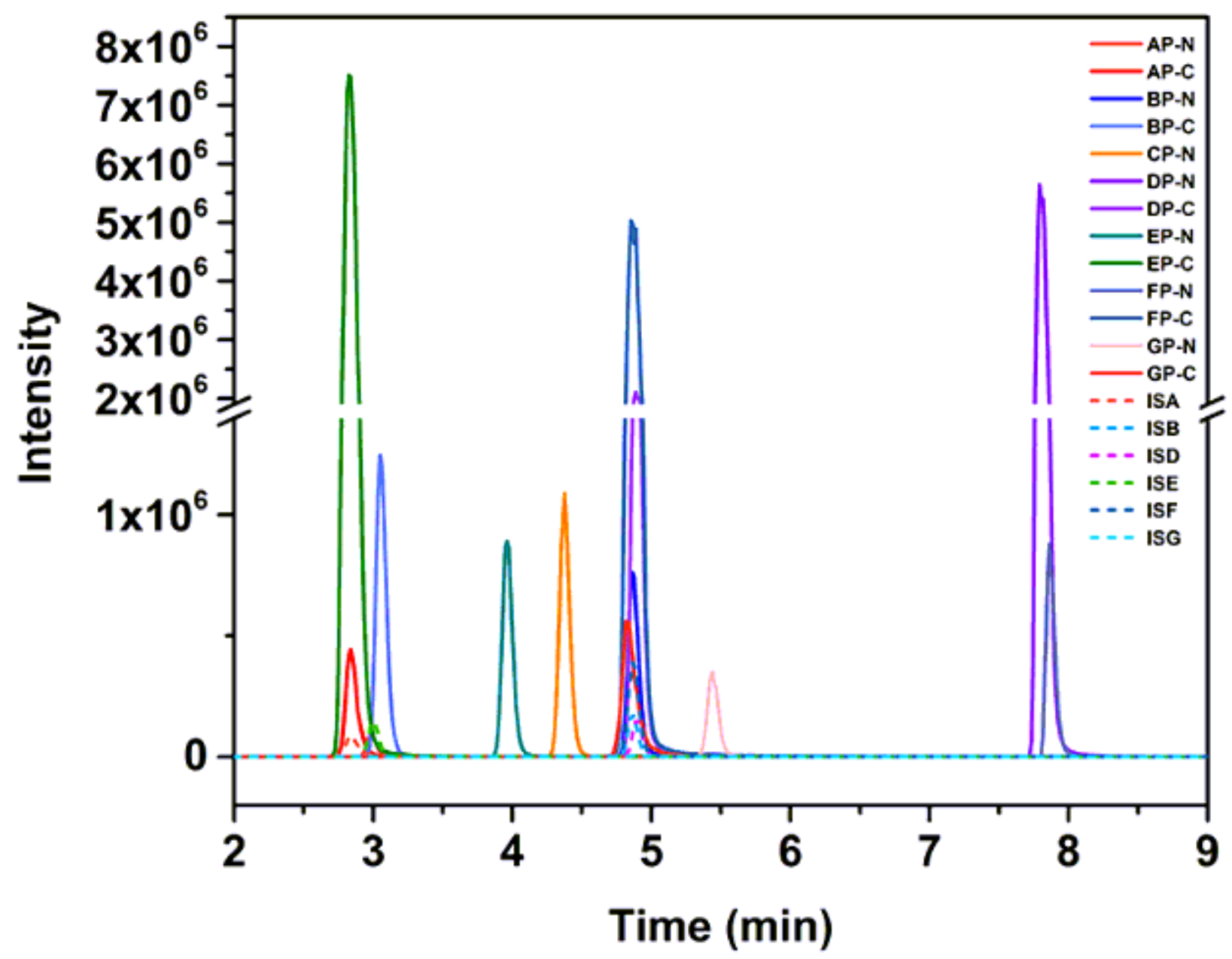

Page 16/18 
Figure 1

LC-MS/MS (MRM) peak intensities for $\mathrm{N}$ - and $\mathrm{C}$-terminal cleavage products and their respective internal standard peptides of BoNT A-G. Synthetic cleavage products of BoNT A to G presented were at concentrations in the middle of their respective linear ranges.

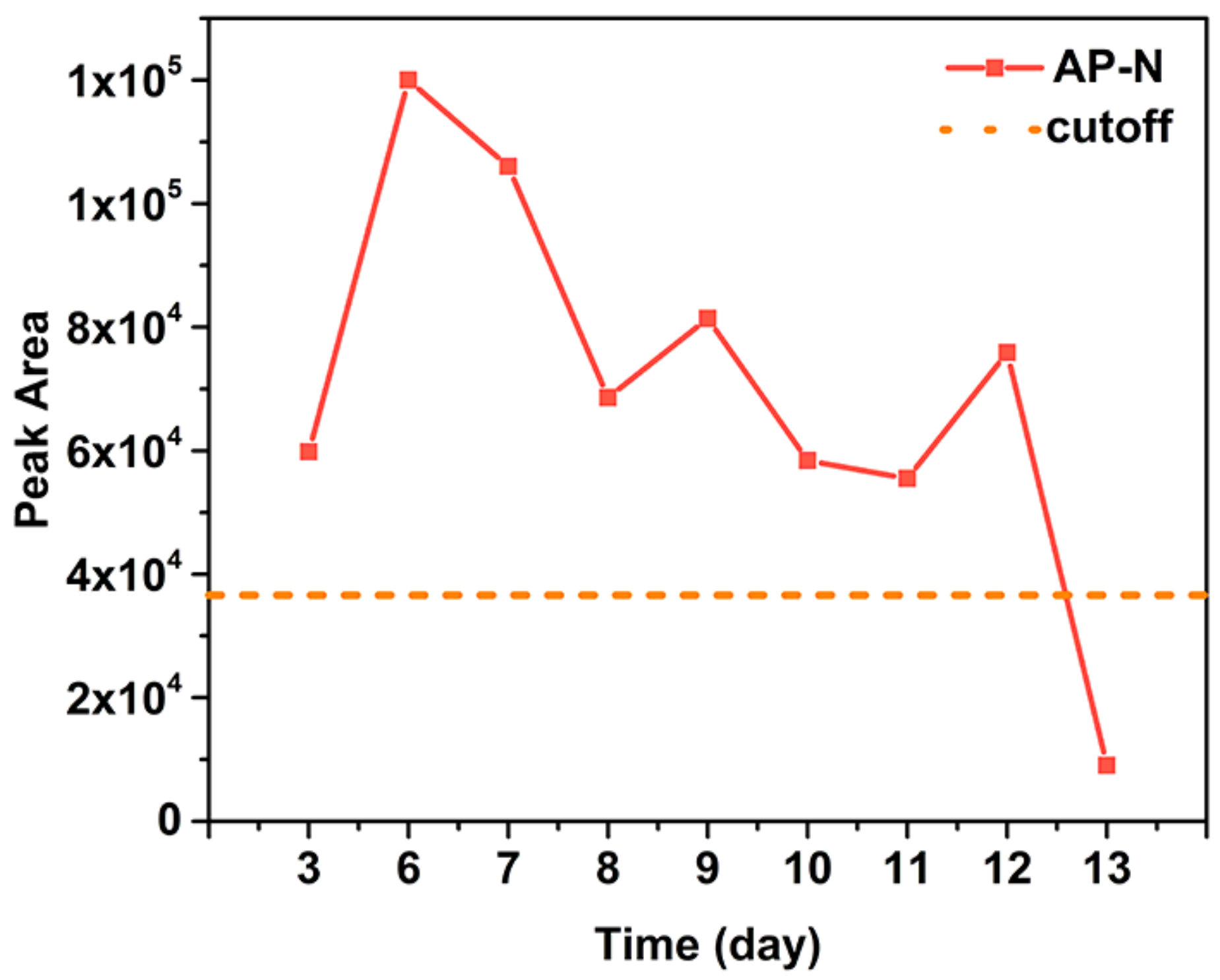

Figure 2

The peak areas of AP-N in sera of patient 2 on different days and the cutoff value (three times of the blank). Results showed sera of patient 2 were positive for BoNT/A on day 3 to day 12 with a maximum on day 6 and then gradually decreased. 


\section{Figure 3}

LC-MS/MS (MRM) peak intensities for N-terminal products of BoNT/A, B and $E$ in clinical samples. a Nterminal products obtained in blank serum. $\mathbf{b}$ N-terminal products obtained in serum samples of patient 2 on day 3 after symptom onset. The results showed serum of patient 2 was positive for BoNT/A, and negative for BoNT/B and E. $\mathbf{c} \mathrm{N}$-terminal products obtained in blank urine. $\mathbf{d} \mathrm{N}$-terminal products obtained in urine samples of patient 1 on day 3 . The results showed urine was positive for BoNT/A, B and $E$ simultaneously. e N-terminal products obtained in vomitus sample of patient 1 on day 3 . The results showed vomitus was positive for BoNT/A, B and E simultaneously. $f \mathrm{~N}$-terminal products obtained in gastric mucosa sample of patient 4 . The results showed the sample was positive for BoNT/E, and negative for BoNT/A and B.

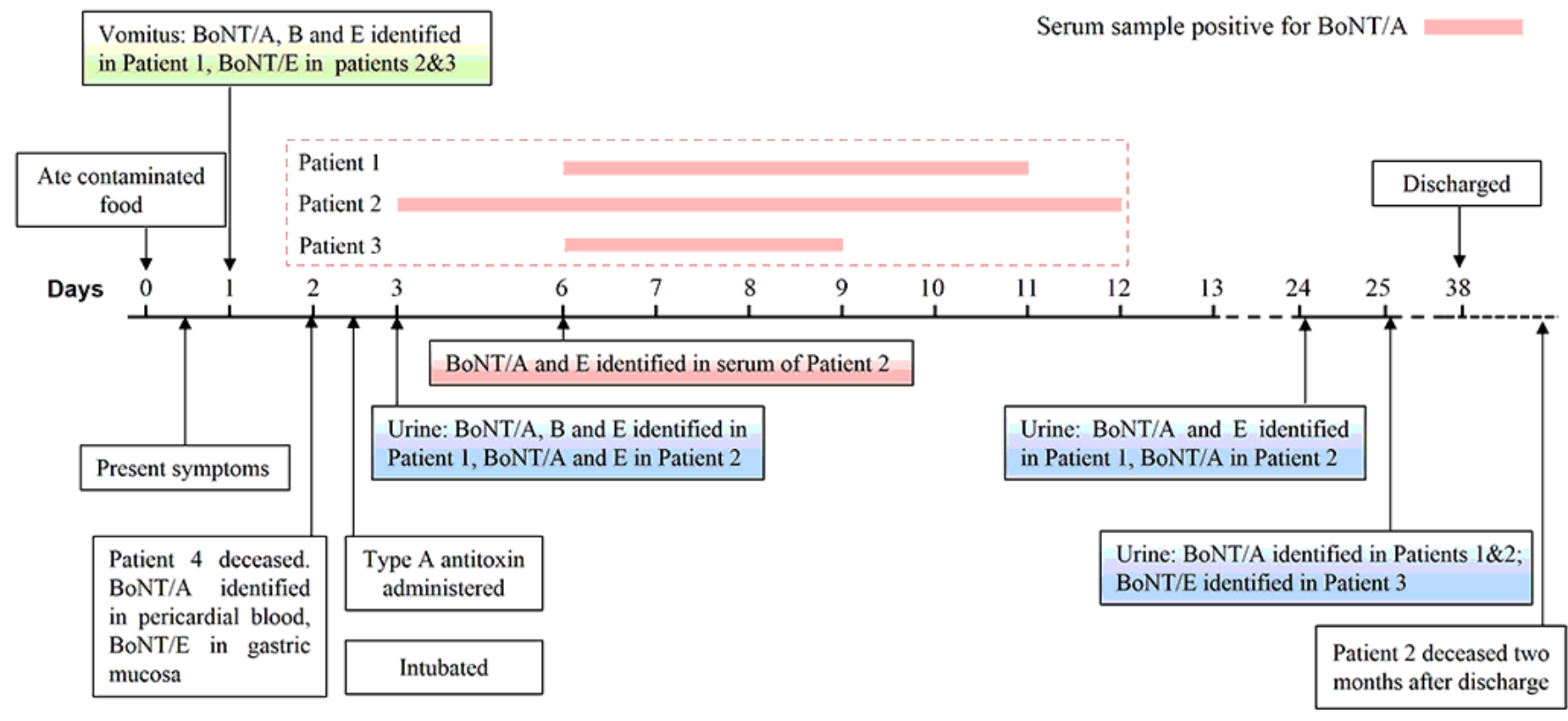

Figure 4

Mixed serotypes A, B and E botulism case timeline.

\section{Supplementary Files}

This is a list of supplementary files associated with this preprint. Click to download.

- Additionalfile.docx 